\title{
New time delay estimation-based virtual decomposition control for n-DoF robot manipulator
}

\author{
Hachmia Faqihi $^{1}$, Khalid Benjelloun ${ }^{2}$, Maarouf Saad $^{3}$, Mohammed Benbrahim $^{4}$, M.Nabil Kabbaj ${ }^{5}$ \\ 1,2EPTICAR, Ecole Mohammadia d'Ingénieurs, Mohammed V University, Rabat, Morocco \\ ${ }^{3}$ Department of Electrical Engineering, Ecole de Technologie Supérieure, Montreal, Canada \\ ${ }^{4,5}$ LIMAS, Faculty of Sciences, Sidi Mohamed Ben Abdellah University, Fez, Morocco
}

\begin{tabular}{|c|c|}
\hline Article Info & ABSTRACT \\
\hline Article history: & \multirow{10}{*}{$\begin{array}{l}\text { One of the most efficient approaches to control a multiple degree-of-freedom robot } \\
\text { manipulator is the virtual decomposition control (VDC). However, the use of the re- } \\
\text { gressor technique in the conventionnal VDC to estimate the unknown and uncertaities } \\
\text { parameters present some limitations. In this paper, a new control strategy of n-DoF } \\
\text { robot manipulator, refering to reorganizing the equation of the VDC using the time } \\
\text { delay estimation (TDE) have been investigated. In the proposed controller, the VDC } \\
\text { equations are rearranged using the TDE for unknown dynamic estimations. Hence } \\
\text { the decoupling dynamic model for the manipulator is established. The stability of the } \\
\text { overall system is proved based on Lyapunov theory. The effectiveness of the proposed } \\
\text { controller is proved via case study performed on 7-DoF robot manipulator and com- } \\
\text { pared to the conventionnal Regressor-based VDC according to some evalution criteria } \\
\text { The results carry out the validity and efficiency of the proposed time delay estimation- } \\
\text { based virtual decomposition controller (TD-VDC) approach. }\end{array}$} \\
\hline Received Feb 16, 2021 & \\
\hline 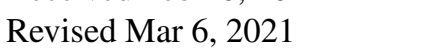 & \\
\hline Accepted Apr 9, 2021 & \\
\hline Keywords: & \\
\hline Free-regressor & \\
\hline Non linear & \\
\hline Robot manipulator & \\
\hline delay estimatio & \\
\hline & \\
\hline
\end{tabular}

This is an open access article under the CC BY-SA license.

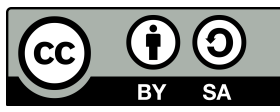

\section{Corresponding Author:}

Hachmia Faqihi

Department of Electrical and Computer Engineering, EPTICAR

Ecole Mohammadia d'Ingénieurs

Mohammed V University, Rabat, Morocco

Email: fhachmia@gmail.com

\section{INTRODUCTION}

In many robotic applications, the principal technical challenges arise in control implementations, specialy when its subject to hight number of degree-of-freedom (DoF). Indded, the robotic systems with high DoF, can be modeled by a set of coupled highly nonlinear differential equations, with various uncertainties and disturbances, which increases the complexity of their control.

A wide range of approaches have been proposed in the literature to control robot systems, ranging from linear to nonlinear techniques, such as computed torque control (CTC), robust control, passivity based control, Lyapunov stability based robust control, sliding mode control (SMC) [1]-[7], Feedback Linearization , Backstepping [8]-[12]. All these techniques are based on the traditional Lagrange-Euler formulation, which present inherent inconvenient, in complexity, then in computational burden [13]-[18].

More we have a high number of DoF in the system, more than the complexity of the dynamic model and the computation burden will increase [19], [20]; It was proportional to the fourth power of the DoF of the robot [21]. This problem limits the use of these algorithms and reduces the feasibility of the control system.

Hence, to cope with the aforementioned problem, a novel theory based on virtual decomposition con- 
trol (VDC) was proposed by [21] to solve the modeling and control problems of a multi-DoF robotic system. It is defined as adaptatif control approach [22]-[25]. In the VDC approach the entire system is decomposed virtually into subsystems ( single joint and single link). The dynamic interaction between two adjacent subsystems is handled by virtual power flow (VPF), which leads to prove the virtual stability. For each subsystem, a subcontroller is designed independently, while the stability of the global system is rigorously maintained [22], [24], [26]-[30]. The obtained dynamic equation of each subsystem is relatively simple, which can reduce the computation burden of the system. Compared to the Lagrangian dynamic model, the computation of VDC method is proportional only to the number of subsystems (DoFs). In order to define the full dynamics of the investigated robot, the VDC approach is based on regression technique. Indeed, the dynamic equation of each subsystem can be linearly parameterized in terms of a regressor matrix, and a unknown parameters vectors, using the required linear/angular velocity vector and its time-derivative. However, the regressor-based VDC control presents difficulties in practical implementation due to the complexity of the regressor matrix, especially to estimate the uncertainties parameters [31], [32]. Indeed, the regressor matrix is known to be highly nonlinear, and its derivation is not unique and remain tedious, although the process is standardized which increase the computation complexity.

In order to avoid the use of the regressor techniques, some alternative tools have been proposed in the literature [33]-[36], using the approximation-based adaptive control based on function approximation technique (FAT). In order to approximate the uncertainties parameter vectors of the dynamic model, the FAT technique is based on linear parameterization in the form of the weighting matrix and the (orthogonal) basis function matrix of the target matrix/vector. However, it presents some limitations. Indeed, the FAT is selected arbitrary without specific criteria, thus an approximation error can be produced. Furthermore, the estimation of the initial values of the weighting matrix is complex. Using the FAT for high DoF can be generated with a computational complexity of weighing and orthogonal matrices [33]. In order to propose a suitable solution to the aforementioned problems, this paper introduces a new nonlinear adaptive control strategy including the virtual decomposition control (VDC) [21] and time delay estimation (TDE) [37], known with its several advantages. The basic idea of the proposed time delay estimation-based virtual decomposition controller (TD-VDC) can be summarized as:

a. The VDC is used as an efficient tool to handle the full-dynamics-based control problem of n-DoF robot manipulator. This approach considers the dynamics of subsystems (rigid bodies and joints) to carry out a tracking trajectory, while guaranteeing the stability and convergence of the entire robotic system.

b. The TDE is used to estimate simply and effectively, the unknown parameters vectors including uncertainties and external disturbances. It require a use of time-delayed information of the control torque inputs and state derivatives for each subsystems.

c. Based on the aforementioned advantages of VDC and TDE approachs, the TDVDC is used to provide an adaptive control with higher precision, ensuring then low computational burden, suitable for high Dof robotic systems.

The remainder of this paper is organized as follows: in Section 1, the dynamic model of each subsystem of n-DOF robot manipulators are described. In Section 3, the proposed TDVDC controller is designed, and compared to the conventional regressor-based virtual decomposition control is presented. Ine Section IV he proposed TDVDC is designed with stability analysis. Section V, a case study is performed on 7-DoF robot manipulator with TDVDC, and compared to the regressor-based VDC. The conclusions are summarized in Section VI.

\section{SYSTEM MODELING}

\subsection{Desription of system}

The equation of motion of an n-DOF robot manipulators are described according to the Euler-Lagrange theory [3], as (1):

$$
\tau=M(q) \ddot{q}+C(q, \dot{q})+G(q)
$$

where $M(q), C(q, \dot{q}), G(q)$ and $G(q, \dot{q})$ are respectively the manipulator's mass matrix, the Coriolis and centrifugal terms vector, the gravity terms vector, and the torque friction vector. In the VDC controller, the dynamic equations of the system can be expressed as link subsystems and joint subsystems, where Virtual Power Flow (VPF) [21] is used to characterize the coupling dynamic interactions among subsystems.

The Figure 1 represents the virtual decomposition of serial robot manipulator to $i$ links, where $\mathrm{i}=$ 
$1, \ldots, \mathrm{n}$, connected via mechanical joints. Each link has one driving cutting point according to the frame $B_{i+1}$ and one driven cutting point according to the frame $B_{i}$. The $i^{t h}$ joint has one driven cutting point according to the frame $B_{i}$ and one driving cutting point according to the frame $T_{i}$. The dynamic equation of every subsystem is derived with respect to the local frame $B_{i}$ according to the Denavit Hartenberg formalism.

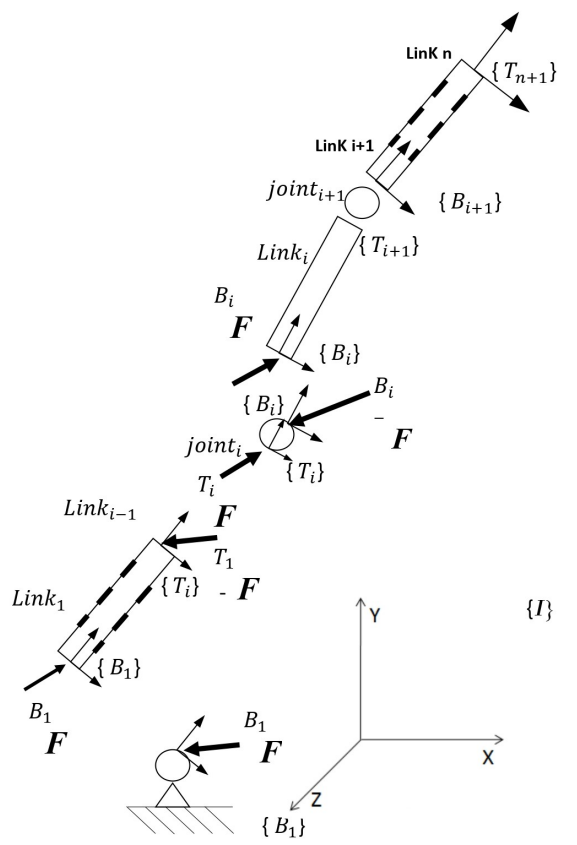

Figure 1. Virtual decomposition shematic of serial robot manipulator [21]

\subsection{Link dynamics}

The dynamic equation of the $i^{t h}$ rigid-link subsystem following its fixed frame can be expressed as (2) $[21]:$

$$
{ }^{B_{i}} F^{*}=M_{B_{i}} \frac{d}{d t}\left({ }^{B_{i}} V\right)+C_{B_{i}}^{B_{i}} V+G_{B_{i}}
$$

where ${ }^{B_{i}} F^{*}$ denote the net force/moment vectors applied from the lower $(i-1)^{t h}$ link to the $i^{\text {th }}$ link expressed in frame $B_{i} .{ }^{B_{i}} V$ is the generalized linear/angular velocity, and $M_{B_{i}}, C_{B_{i}}, G_{B_{i}}$ represent respectively the inertial, Centrifugal/Coriolis, and gravitational terms, respectively. Using an iterative process computation, the vector of the total generalized force (forces/moments) acting on the $i^{t h}$ rigid body can be computed as (3):

$$
\begin{array}{r}
{ }^{B_{n}} F={ }^{B_{n}} F^{*} \\
{ }^{B_{n-1}} F={ }^{B_{n-1}} F^{*}+{ }^{B_{n-1}} U_{B_{n}}{ }^{B_{n}} F^{*} \\
. \\
. \\
{ }^{B_{i}} F={ }^{B_{i}} F^{*}+{ }^{B_{i}} U_{B_{i+1}}{ }^{B_{i+1}} F^{*}
\end{array}
$$

where ${ }^{B_{i}} F$ is the generalized force exerted by body $i+1$ on body $i,{ }^{B_{i+1}} F$ is the generalized force exerted by body $i+1$ on body $i,{ }^{B_{i}} V_{r}$ is the velocity of body $i .{ }^{B_{i}} U^{T} B_{i+1}$ is the transformation matrix, defined as (4):

$$
{ }^{B_{i}} U^{T}{ }_{B_{i+1}}=\left[\begin{array}{cc}
{ }^{B_{i}} R_{B_{i+1}} & 0_{3 x 3} \\
S\left({ }^{B_{i}} r_{B_{i+1}}\right)^{B_{i}} R_{B_{i+1}} & { }^{B_{i}} R_{B_{i+1}}
\end{array}\right]
$$

where ${ }^{B_{i}} R_{B_{i+1}}$ represents the rotation matrix from frame $B_{i}$ to the frame $B_{i+1}, 0_{3 x 3}$ is the null matrix, $S$ is the skew-symmetric matrix operator performing the cross product between two vectors, and ${ }^{B_{i}} r_{B_{i+1}}$ denotes a vector from the origin of frame $B_{i}$ to the frame $B_{i+1}$, expressed in frame $B_{i}$. 


\subsection{Joint dynamics}

The dynamic equation of the $i^{\text {th }}$ joint subsystem expressed in its fixed frame is given by the following (5) $[21]:$

$$
\tau_{i j}=I_{m i} \ddot{q}_{i}+k_{c i} \operatorname{sign}\left(\dot{q}_{i}\right)
$$

where $I_{m i}$ and $k_{c i}$ denote the moment of inertia, and the Coulomb friction coefficient of the $i^{\text {th }}$ joint respectively.

Finally the control torque of the global system can be expressed by (6):

$$
\tau_{i}=\tau_{i j}+\tau_{i l}
$$

$\tau_{i j}$ and $\tau_{i}$ denote the net torque and the control torque applied to the $i^{\text {th }}$ joint respectively. $\tau_{i l}$ denotes the output torque of the $i^{\text {th }}$ joint toward the links, which can be computed in magnitude by the torque projected from the links, expressed in (7).

$$
\tau_{i l}=z^{T B_{i}} F
$$

where $z=[0,0,0,0,0,1]^{T}$ for revolute joint. $\dot{q}_{i}$ the joint velocity vector.

\section{CONTROLLER DESIGN}

The main control objective in VDC approach is to track a required trajectory such that the joint tracking error between the actual and required velocity converges asymptotically to zero in finite-time, with high accuracy even in presence of uncertainties and external disturbances.

In order to design the VDC controller some vectors must be defined related to this approach.

\subsection{Required vectors}

The required velocity, is one of the concept related to VDC approach [21], wich can be expressed as

$$
\dot{q}_{r}=\dot{q}_{d}+\lambda\left(q_{d}-q\right)
$$

$\dot{q}_{d}$ and $q_{d}$ denote respectively the desired joint velocity and the desired joint angle, $\lambda>0$ is a constant.

The dynamic equations for the VDC controller design are based on the required joint velocity and the required linear/angular velocity vectors.

The required linear/angular velocity of the link can be computed as (9):

$$
{ }^{B_{i+1}} V_{r}=z \dot{q}_{(i+1) r}+{ }^{B_{i}} U^{T}{ }_{B_{i+1}}{ }^{B_{i}} V_{r}
$$

Adaptive control law of rigid-link subsystem

\subsection{Adaptive control law of link subsystem}

Referring to the dynamic link subsystem (2), and the required linear/angular velocity vector and its time-derivative, the required force/moment vectors are expressed as (10) [21]:

$$
{ }^{B_{i}} F_{r}{ }^{*}=M_{B_{i}} \frac{d}{d t}\left({ }^{B_{i}} V_{r}\right)+C_{B_{i}}{ }^{B_{i}} V_{r}+G_{B_{i}}
$$

Where ${ }^{B_{i}} F_{r}{ }^{*}$ the required net force/moment vectors of the subsystem links.

${ }^{B_{i}} V$ the vector of the generalized velocities (i.e., linear and angular components), wich can be expressed as (11):

$$
{ }^{B_{i}} V=z \dot{q}_{i}+{ }^{B_{i-1}} U^{T}{ }_{B_{i}}{ }^{B_{i-1}} V
$$

Consider the linear parameterization form, the link subsystem (2) can be expressed as (12):

$$
{ }^{B_{i}} F_{r}{ }^{*}=Y_{l i} \theta_{l i}
$$

where the $Y_{l i}$ denotes the $i^{\text {th }}$ regressor matrix formed by the joint velocity, the linear/angular velocity and its time-derivative; and $\theta_{l i}$ denotes the $i^{t h}$ parameters vector formed by the uncertainties parameter vector. Therefore, the control law of link subsystem is designed as (13):

$$
{ }^{B_{i}} F_{r}{ }^{*}=Y_{l i} \hat{\theta}_{l i}+K_{l i}^{B_{i}} e_{V}
$$


where $K_{l i}$ is a diagonal matrix representing the gain of the feedback controller, and ${ }^{B_{i}} e_{V}$ is a measure of the tracking accuracy defined by (14):

$$
{ }^{B_{i}} e_{V}={ }^{B_{i}} V_{r}-{ }^{B_{i}} V
$$

$\hat{\theta}_{l i}$ is the estimate of the uncertainties parameter vector $\theta_{l i}$ Finally, the control law of link subsystem, can be computed by an iterative process, as (15):

$$
\begin{array}{r}
{ }^{B_{n}} F_{r}=Y_{l n} \hat{\theta}_{l n}+K_{l n}{ }^{B_{n}} e_{V} \\
. \\
{ }^{B_{i}} F_{r}=Y_{l i} \hat{\theta}_{l i}+K_{l i}{ }^{B_{i}} e_{V}+{ }^{B_{i}} U_{B_{i+1}}{ }^{B_{i+1}} F_{r}{ }^{*}
\end{array}
$$

\subsection{Adaptive control law of joint subsystem}

For the control law of joint subsystem defined as, the required net torque $\tau_{i j r}$ applied to the $i^{t h}$ joint, is based on the required joint velocity vectors, as (16) [21]:

$$
\tau_{i j r}=I_{m i} \ddot{q}_{i r}+k_{c i} \operatorname{sign}\left(\dot{q}_{i r}\right)
$$

According to the linear parameterization property, the required net torque $\tau_{i r}^{*}$ can be written in linear form as (17):

$$
\tau_{i j r}=Y_{j i} \theta_{j i}
$$

where $Y_{j i}$ denotes the regressor matrix formed by the joint velocity and acceleration, and $\theta_{j i}$ denotes the parameters vector formed by the physical dynamic parameters.

Due to the difficulty in knowing the exact value of the physical parameters of the $i^{\text {th }}$ joint, they should be estimated. Then the estimation vector denoted by $\hat{\theta}_{j i}$ is used, and the equation of control becomes (18):

$$
\tau_{i j r}=Y_{j i} \hat{\theta}_{j i}+K_{j i} e_{q}
$$

where $K_{j i}$ is a diagonal matrix representing the gain of the feedback controller, and $e_{q}$ is a tracking joint error defined by (19):

$$
e_{q i}=\dot{q}_{i r}-\dot{q}_{i}
$$

Finally, the total control torque is computed using the required output torque of the $i^{\text {th }}$ joint toward the links, and the required control torque of the $i^{\text {th }}$ joint as (20):

$$
\tau_{i}=\tau_{i j r}+\tau_{i l r}
$$

where $\tau_{i j r}$ denotes the control torque of the $i$ th joint, and $\tau_{i l r}$ the required output torque of the $i$ th joint toward the links expressed with the required force/moment vectors as (21):

$$
\tau_{i l r}=z^{T B_{i}} F_{r}
$$

The control based VDC approach is to resolve equation (20), where the vectors of parameters estimation $\hat{\theta}_{l i}$ and $\hat{\theta}_{j i}$ are used. The parameter adaptation function should be chosen to ensure system stability.

\subsection{Regressor-based VDC controller}

In the conventionnal Regressor-based VDC controller, the uncertainties parameter vectors $\hat{\theta}_{j i}$ and $\hat{\theta}_{l i}$ for joint and link subsystems, are updated using the projection function $P$ defined as a differentiable scalar function [21]. According to the link subsystem, the uncertainties parameter vector is estimated as (22):

$$
\hat{\theta}_{j i \gamma}=P\left(s_{i \gamma}(t), \rho_{i \gamma}, a_{i \gamma}(t), b_{i \gamma}(t), t\right)
$$

where $\hat{\theta}_{j i \gamma}$ denotes the $\gamma$ th element of $\hat{\theta}_{j i}, s_{i \gamma}(t)$ denotes the $\gamma$ th element of $s_{i}(t)$ defined as (23) [21]:

$$
s_{i}(t)=Y_{j i}^{T}\left({ }^{B_{i}} V_{r}-{ }^{B_{i}} V\right)
$$

Int J Rob \& Autom, Vol. 10, No. 3, September 2021 : $192-206$ 
$\rho_{i \gamma}>0$ is a parameter update gain, and $a_{i \gamma}(t), b_{i \gamma}(t)$ denote the lower and upper bounds of $\theta_{j i \gamma}$.

The projection function $P$ is a differentiable scalar function defined by its time derivative which is governed by (24) and (25):

$$
\dot{P}(t)=\rho s(t) \kappa
$$

with

$$
\kappa=\left\{\begin{array}{c}
0 \text { if } P(t)<a(t) \text { and } s(t)<0 \\
0 \text { if } P(t)>b(t) \text { and } s(t)>0 \\
1 \text { otherwhise }
\end{array}\right.
$$

It is the same for $\hat{\theta}_{l i}$ of the required net torque.

The use of the projection function for the uncertainties parameter vectors estimation, requires to compute the derivative of the regressor matrix in every sampling cycle. However, the regressor matrix derivation is not unique, though the process is standardized. Furtheremore, it presents high complexity, then an additionnal computational burden

\subsection{Proposed TDVDC controller}

As demonstrated, regressor-based VDC method presents inherent limits regarding the use of the projection function to estimate the unknown parameter vectors $\hat{\theta}_{l i}$ and $\hat{\theta}_{j i}$ for link and joint subsystems respectively. To over come the issues with regressor-based VDC technique, a new control strategy combining the TDE and VDC approachs is proposed. The idea is refered to estimate the dynamic uncertainties and parameter vectors by the use of TDE. Refering to the dynamic link subsystem given in the (2), and the dynamique joint subsystem given in the (5), the dynamic uncertainties and unknown parameter vectors can be regrouped as (26) and (27):

For the link subsystems

$$
{ }^{B_{i}} F^{*}=M_{B_{i}} \frac{d}{d t}\left({ }^{B_{i}} V\right)+H_{l i}
$$

For the Joint subsystems

$$
\tau_{i j r}=I_{m i} \ddot{q}_{i r}+H_{j i}
$$

$H_{l i}$ and $H_{j i}$ represents the dynamic uncertainties and unknown parameter vectors of the link and joint subsystems respectively, where (28) and (29):

$$
\begin{array}{r}
H_{l i}=C_{B_{i}}{ }^{B_{i}} V_{r}+G_{B_{i}} \\
H_{j i}=k_{c i} \operatorname{sign}\left(\dot{q}_{i r}\right)
\end{array}
$$

Therefore, the control law subsystems, are given by (30):

$$
\left\{\begin{array}{r}
{ }^{B_{i}} F_{r}{ }^{*}=\bar{m}_{i}{ }^{B_{i}} \dot{V}+\hat{H}_{l i}+K_{l i}{ }^{B_{i}} e_{V} \\
\tau_{i j r}=\bar{i}_{i} \ddot{q}_{i r}+\hat{H}_{j i}+K_{j i} e_{q i}
\end{array}\right.
$$

$\bar{m}_{i}$ and $\bar{i}_{i}$ are a constant coefficients associated to $M_{B_{i}}$, and $I_{m i}$ respectively. The determination of both constant coefficients $\bar{m}_{i}$ and $\bar{i}_{i}$ is discussed in [37], [38]. $\hat{H}_{l i}$ and $\hat{H}_{j i}$ represents respectively the estimate of $H_{l i}$ and $H_{j i}$. 
In order to design the TDVDC controller and carry out its stability analysis, let consider the following assumptions:

A1: The joint position and velocity are measured.

A2: The parameter vectors $H_{l i}$ and $H_{j i}$

their time derivatives $\frac{d}{d t} H_{l i}$ and $\frac{d}{d t} H_{j i}$ are globally Lipschitz functions.

A3: The constant coefficients $\bar{m}_{i}$ and $\bar{i}_{i}$ are chosen assuming that:

$$
\begin{array}{r}
\left\|I_{n}-M(q) \bar{m}^{-1}\right\|<1 \\
\left\|I_{n}-I(q) \bar{i}^{-1}\right\|<1
\end{array}
$$

According to the use of TDE [37], and if the assumption A2 is verified, we can estimate $H_{l i}$ and $H_{j i}$. Indeed the value of the function $H_{l i}$ and $H_{j i}$ are considered at the present time $t$, very close to that at time $(t-T)$ in the past for a small time delay $T$ in (31).

For the link subsystem

$$
\hat{H}_{l i}(t) \cong \hat{H}_{l i}(t-T)
$$

therefore, using an iterative process, the estimate of the uncertainties parameter vector of the link substem $\hat{H}_{l i}(t)$ can be computed as:

$$
\left\{\begin{array}{r}
\hat{H}_{l i}(t) \simeq \tau_{i l r}(t-T) z-\bar{m}_{i}{ }^{B_{i}} \dot{V}(t-T)-K_{l i}{ }^{B_{i}} e_{V}(t-T)- \\
{ }^{B_{i}} U_{B_{i+1}}(t-T) \hat{H}_{l(i+1)}(t-T)+\bar{m}_{i+1}{ }^{B_{i+1}} \dot{V}(t-T)+ \\
K_{l(i+1)}{ }^{B_{i+1}} e_{V}(t-T) \\
\hat{H}_{l(i+1)}(t) \simeq\left(\tau_{(i+1) l r}(t-T) z-\bar{m}_{i+1}{ }^{B_{i+1}} \dot{V}(t-T)-\right. \\
K_{l i+1}{ }^{B_{i+1}} e_{V}(t-T)-{ }^{B_{i+1}} U_{B_{i+2}}(t-T) \hat{H}_{l(i+2)}(t-T)+ \\
\bar{m}_{i+2}{ }^{B_{i+2}} \dot{V}(t-T)+K_{l(i+2)}{ }^{B_{i+2}} e_{V}(t-T) \\
\hat{H}_{l n}(t) \simeq \tau_{n l r}(t-T) z-\bar{m}_{n}{ }^{B_{n}} \dot{V}(t-T)-K_{l n}{ }^{B_{n}} e_{V}(t-T)
\end{array} .\right.
$$

For the joint substem, the estimates of the uncertainties parameter vector $\hat{H}_{j i}(t)$ is given by (32) and (33):

$$
\hat{H}_{j i}(t) \cong \hat{H}_{j i}(t-T)
$$

then

$$
\hat{H}_{j i}(t) \cong \tau_{i j r}(t-T)-K_{j i} e_{q i}(t-T)
$$

where $T$ is the estimation time delay. The accuracy estimation of $\hat{H}_{l i}(t)$ and $\hat{H}_{j i}(t)$ improves for a small $T$. In practice, the smallest estimation time delay $T$ is chosen to be the sampling period which means that the perfect parameters vector are identified every sampling period. Finally, the proposed control is obtained as (34)-(36):

$$
\tau_{i}(t)=\tau_{i j r}(t)+\tau_{i l r}(t)
$$

where

$$
\tau_{i j r}(t)=\bar{i}_{i} \ddot{q}_{i r}(t)+\hat{H}_{j i}(t)+K_{j i}\left(\dot{q}_{i r}(t)-\dot{q}_{i}(t)\right)
$$

and

$$
\begin{aligned}
& \tau_{i l r}(t)=z^{T}\left[\bar{m}_{i}{ }^{B_{i}} \dot{V}(t)+\hat{H}_{l i}(t)+K_{l i}{ }^{B_{i}} e_{V}(t)-\right. \\
& \left.{ }^{B_{i}} U_{B_{i+1}}\left[\bar{m}_{i+1}{ }^{B_{i+1}} \dot{V}(t)+\hat{H}_{l(i+1)}(t)+K_{l(i+1)}{ }^{B_{i+1}} e_{V}(t)\right]\right]
\end{aligned}
$$

The closed-loop control system based on the proposed TDVDC technique is presented in Figure 2.

Int J Rob \& Autom, Vol. 10, No. 3, September 2021 : $192-206$ 


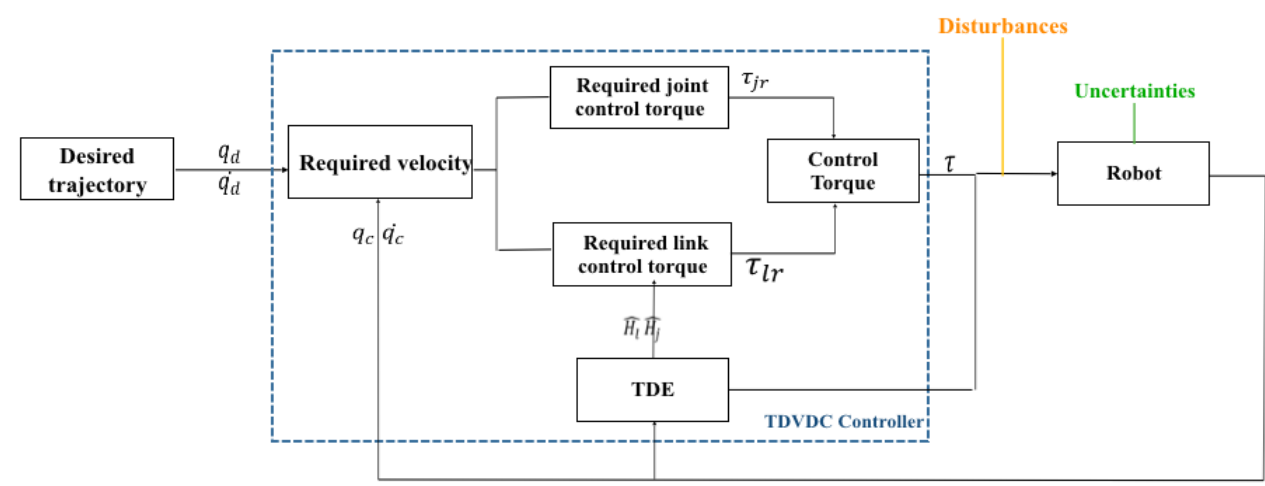

Figure 2. Block diagram of the proposed controller

\section{VIRTUAL STABILITY ANALYSIS}

According to the virtual work approach, the global stability of the system's VDC is proven through the virtual stability of each subsystem. Indeed, using the definition 2.17 and theorem 2.1 in [21], the global system is stable in the sense of Lyapunov, if each subsystem is proved to be virtually stable. It will be proven that all the decomposed subsystems of the studied system with their respective control equations are virtually stable, leading to the stability of the entire system. Generally, the stability analysis, in the sense of the Lyapunov approach, refers to define a positive candidate function and then to show that its variation is a decreasing function. Considering the Lyapunov candidate function for the entire robot as summation of two functions for the link $\left(V_{l i}\right)$ and joint $\left.V_{j i}\right)$ subsystems as $(37): i=\{0, \ldots, n\}$

$$
V=\sum_{i} V_{l i}+\sum_{i} V_{j i}
$$

\subsection{Virtual stability of the ith link}

Let consider the Lyapunov candidate function for the $i^{\text {th }}$ link as (38):

$$
V_{l i}=\frac{1}{2}{ }^{B_{i}} e_{V}{ }^{T} M_{B_{i}}{ }^{B_{i}} e_{V}+\frac{1}{2}\left(H_{l i}-\hat{H}_{l i}\right)^{2}
$$

Then from [21] and the dynamic equation of the $i^{\text {th }}$ link given in (13), the first derivative along time of $V_{l i}$ can be given by (39):

$$
\begin{array}{r}
\dot{V}_{l i}=-{ }^{B_{i}} e_{V}{ }^{T} K_{l i}{ }^{B_{i}} e_{V}+{ }^{B_{i}} e_{V}{ }^{T}\left({ }^{B_{i}} F_{r}-{ }^{B_{i}} F\right)+ \\
\left(H_{l i}-\hat{H}_{l i}\right)\left({ }^{B_{i}} e_{V}-\dot{\hat{H}}_{l i}\right)
\end{array}
$$

where ${ }^{B_{i}} e_{V}{ }^{T} C_{B i}{ }^{B_{i}} e_{V}=0$, since $C_{B i}$ defined as skew-symmetric.

According to the TDE use the $\dot{V}_{l i}$ becomes (40):

$$
\begin{array}{r}
\dot{V}_{l i}=-{ }^{B_{i}} e_{V}{ }^{T} K_{l i}{ }^{B_{i}} e_{V}+{ }^{B_{i}} e_{V}{ }^{T}\left({ }^{B_{i}} F_{r}^{*}-{ }^{B_{i}} F_{r}\right)+ \\
\Delta H_{l i}\left(Y_{i}^{T B^{B}} e_{V}-\frac{1}{2 T} \Delta H_{l i}\right)
\end{array}
$$

where $\Delta H_{l i}(t)=H_{l i}(t)-H_{l i}(t-T)$, is the term due to the TDE error.

Otherwise, as $H_{l i}(t)$ is a Lipschitz function, then (41):

$$
\left|\Delta H_{l i}\right| \leq \delta_{l i} T
$$

$\delta_{l i}$ is the Lipschitz constant.

To perform the VDC for each subsystem, the virtual power flows are introduced to characterize the dynamic interaction among the subsystems at its cutting points. Indeed, the virtual power flow is defined as the inner 
product of the linear/angular velocity error vector and the force/moment error vector, with respect to the frame $\{A\}$, as (42):

$$
p_{A}={ }^{A} e_{V}^{T}\left({ }^{A} F_{r}^{*}-{ }^{A} F_{r}\right)
$$

Therefore from [21], (40)-(42), we obtain:

$$
\dot{V}_{l i} \leq-{ }^{B_{i}} e_{V}{ }^{T} K_{l i}{ }^{B_{i}} e_{V}+p_{B_{l i}}-p_{T_{l i}}-\frac{1}{2} \delta_{l i}
$$

where $p_{B_{l i}}$ and $p_{T_{l i}}$ represent the virtual power flows at the two cutting points of each link.

As defined in [21], according to an open chaine structure, for $p_{B_{l 1}}=0$ and $p_{T_{l n}}=0$ the total virtual power flows is given by (44):

$$
\sum_{i}^{n}\left(p_{B_{l i}}-p_{T_{l i}}\right)=0
$$

Therefore the (43) becomes (45):

$$
\sum_{i} \dot{V}_{l i} \leq \sum_{i}\left(-{ }^{B_{i}} e_{V}{ }^{T} K_{l i}{ }^{B_{i}} e_{V}-\frac{1}{2} \delta_{l i}\right)
$$

\subsection{Virtual stability of the ith joint}

The positive Lyapunov candidate function related to the joint dynamics can be chosen according to the joint dynamic and its control law, as (46):

$$
V_{j i}=\frac{1}{2} I_{m i} e_{q}^{2}+\frac{1}{2}\left(H_{j i}-\hat{H}_{j i}\right)^{2}
$$

Then, its time derivative is (47):

$$
\dot{V}_{j i}=-e_{q i} I_{m i} \dot{e}_{q i}-\left(H_{j i}-\hat{H}_{j i}\right) \dot{\hat{H}}_{j i}
$$

with the TDE use, and the dynamic equation of the $i^{t h}$ joint given in (18), the $\dot{V}_{j i}$ becomes (48):

$$
\dot{V}_{j i}=-K_{j i} e_{q i}^{2}+e_{q i}\left(\tau_{i r}^{r}-\tau_{i r}\right)-\frac{1}{2 T} \Delta H_{j i}^{2}
$$

According to [21], (41), (48), and VPF definition, we obtain (49):

$$
\dot{V}_{j i} \leq-K_{j i} e_{q i}^{2}-\frac{1}{2} \delta_{j i}+p_{B_{j i}}-p_{T_{j i}}
$$

As described in the above section, using VFP the (49) becomes (50)

$$
\sum \dot{V}_{j i} \leq \sum\left(-K_{j i} e_{q i}^{2}-\frac{1}{2} \delta_{j i}\right)
$$

\subsection{Stability of the global system}

The derivative of the global Lyapunov candidate function (37), is given as (51):

$$
\dot{V}=\sum \dot{V}_{l i}+\sum \dot{V}_{j i}
$$

The $\dot{V}$ function is proved to be always decreasing based on the virtual power as the inner product of the linear angular velocity vector error and the force moment vector error presented in [21], and the choice of the parameter function adaptation, where (52):

$$
\dot{V} \leq-\sum_{i, j}\left({ }^{B_{i}} e_{V}{ }^{T} K_{l i}{ }^{B_{i}} e_{V}+\frac{1}{2} \delta_{l i}+K_{j i} e_{q i}^{2}+\frac{1}{2} \delta_{j}\right)
$$

where $\delta_{j}>0$ and $\delta_{l}>0$ are the Lipschitz constants. Since $\dot{V}<0$ where all gains are positive, the system is asymptotically stable in the sense of Lyapunov [21].

Int J Rob \& Autom, Vol. 10, No. 3, September 2021 : 192 - 206 


\section{CASE STUDY}

\subsection{Simulation description}

To illustrate the effectiveness of the proposed control strategy, in this section a case study is performed for tracking trajectory of 7-DoF robotic manipulator using the proposed TDVDC. The simulation routine is conducted following the control architecture given in Figure 2, wich includes the desired trajectory given in joint space. The desired joint velocity and the desired joint acceleration are obtained from the derivation of the desired joint position. The equation of motion for each link and joint (i) $(i=1, \ldots, 7)$ subsystems is derived with respect to a local frame $\left\{B_{i}\right\}$ as shown in Figure 1. The mass, Coriolis, and the gravity termes of the link (i) can be described by (53)-(55):

$$
\begin{gathered}
M_{B_{i}}=\left[\begin{array}{ccc}
m_{i} & 0 & 0 \\
0 & m_{i} d_{i} & m_{i} d_{i} \\
0 & m_{i} d_{i} & I_{i}+m_{i} d_{i}^{2}
\end{array}\right], i=1, \ldots, 7 \\
C_{B_{i}}=\left[\begin{array}{ccc}
0 & -m_{i} & -m_{i} d_{i} \\
m_{i} & 0 & 0 \\
m_{i} d_{i} & 0 & 0
\end{array}\right] \dot{q}_{i}, i=1, \ldots, 7 \\
G_{B_{i}}=\left[\begin{array}{c}
m_{i} \sin \left(q_{i}\right) g \\
m_{i} \cos \left(q_{i}\right) g \\
m_{i} d_{i} \cos \left(q_{i}\right) g
\end{array}\right], i=1, \ldots, 7
\end{gathered}
$$

where the physical parameters of the using robot system are represented in Table 1. The numerical simulations are conducted for the proposed TDVDC controller and compared to the conventional regressor-based VDC in order to prove the effectiveness of the prposed approach. During the trajectory tracking, a disturbances was added to the torque input representing $5 \%$ of maximum value of the torque after $t=10 \mathrm{~s}$. In addition an uncertainty function $U(t)$ was injected to the robot dynamic model to validate the effectiveness of the proposed control strategy in (56).

$$
U\left(q_{i}, t\right)=q_{i} * \sin (t)+0.5 * \sin (500 * p i * t)
$$

Table 1. Physical parameters

\begin{tabular}{l}
\hline$l_{1}=0.3 \mathrm{~m} ; l_{2}=0.5 \mathrm{~m} ; l_{3}=l_{4}=0.21 \mathrm{~m} ; l_{5}=0.25 \mathrm{~m} ; l_{6}=0.5 \mathrm{~m}$ \\
\hline$m_{1}=0.122 \mathrm{Kg} ; \mathrm{m}_{2}=0.66 \mathrm{Kg} ; \mathrm{m}_{3}=0.08 \mathrm{Kg} ; \mathrm{m}_{4}=0.175 \mathrm{Kg} ; \mathrm{m}_{5}=0.251 \mathrm{Kg} ; \mathrm{m}_{6}=0.023 \mathrm{Kg}$ \\
$k_{c_{1}}=k_{c_{2}}=k_{c_{3}}=k_{c_{4}}=k_{c_{5}}=k_{c_{6}}=0.5 \mathrm{~N} . \mathrm{m}$ \\
$I_{1}=I_{2}=I_{3}=I_{4}=I_{5}=I_{6}=0.0234 \mathrm{Kg} . \mathrm{m}^{2}$ \\
\hline
\end{tabular}

For the proposed TDVDC approach, the target robot is controlled following the closed-loop given in Figure 2. It concerns the use of TDE for the estimation terms defining the unknown and uncertainties parameter vectors of the robot. The required linear/angular velocity and its time derivative is computed using $\lambda$ constant. The constant coefficients $\bar{m}_{i}$ and $\bar{i}_{i}$ are chosen according to the assumption A3. A suitable choose of these constants influence the stability and attenuation of measurement noise. These constants are conducted by the trial and error method. The time delay $T$ is fixed as sampling time. The gain parameters of the feedback controller $K_{j}$ and $K_{l}$ for joint and link subsystems respectivelyare are fixed ensuring the stability condition. These parameters values must be adjusted in order to obtain the optimum performance. For the conventional regressor-based VDC approach, the parameters estimation is based on projection function presented in (22) which requires the derivation of the regressor matrix in every sampling time, as discussed previously. To accomplish the simulation routine, in addition to the gains feedback controller $K_{j}, K_{l}$ and $\lambda$, the parameters $\rho$, $a, b$ are used for the projection function.

\subsection{Simulation results}

The obtained simulation results of the tracking trajectory and the traking errors for the proposed TDVDC and the conventional Regressor-based VDC strategies are shown in Figure 3, Figure 4, and Figure 5 respectively 
It is shown that the system response converges for the conventional regressor-based VDC and the proposed TDVDC approaches, with insensitivity to uncertainties and disturbances. However, for the proposed TDVDC the signal response present some delay at the begining before $t=0.2 \mathrm{~s}$. The obtained joint torque for the TDVDC and Regressor-based VDC are given respectively in Figure 6 and Figure 7. It should be noted that the feedback gains should carefully be selected to get an accurate tracking.
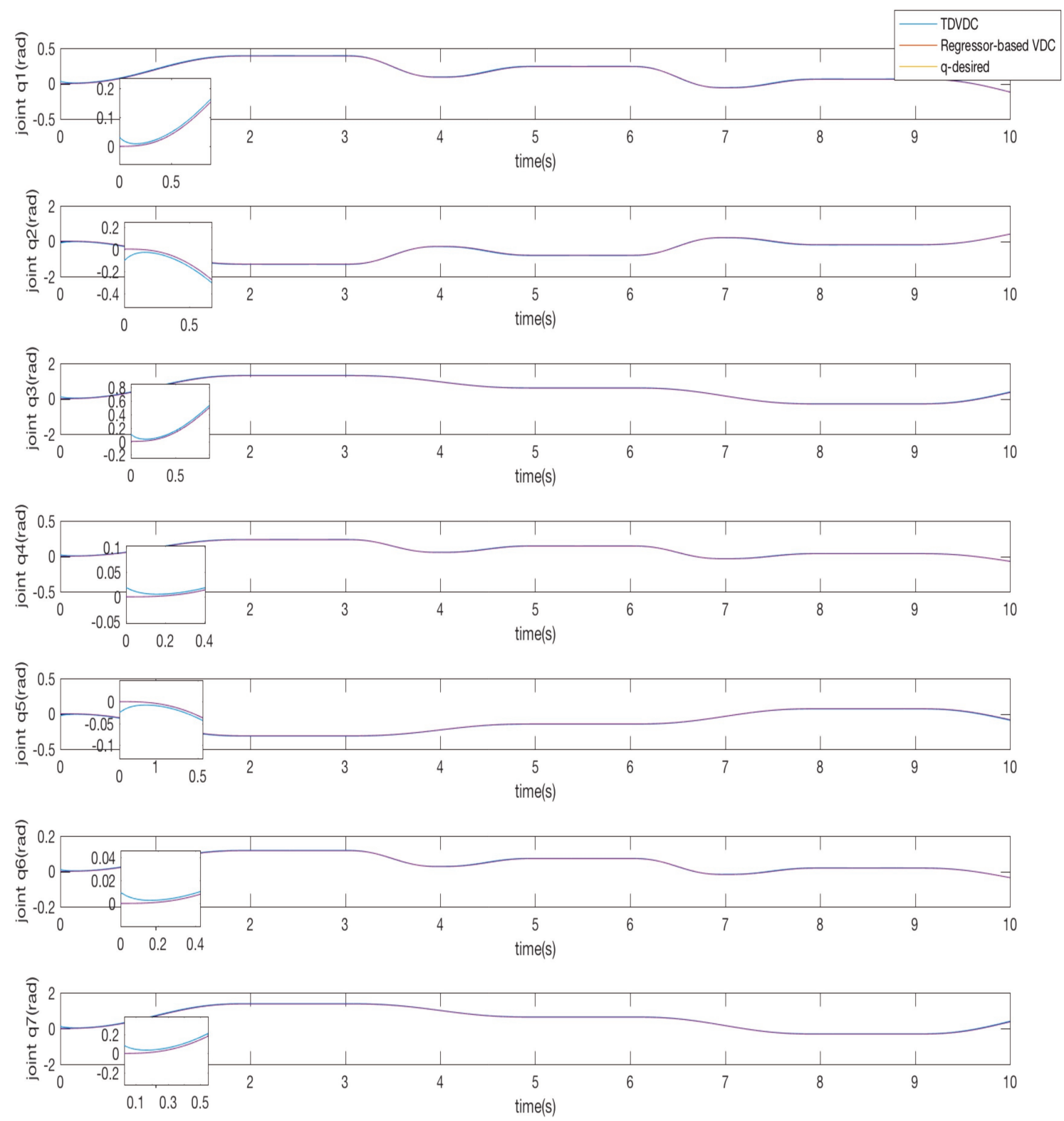

Figure 3. Tracking joint trajectory (a: for the joint $q_{1}$, b: for the joint $q_{2}$, c: for the joint $q_{3}$, d: for the joint $q_{4}$, e: for the joint $q_{5}$, f: for the joint $q_{6}, \mathrm{~g}$ : for the joint $q_{7}$ ) 


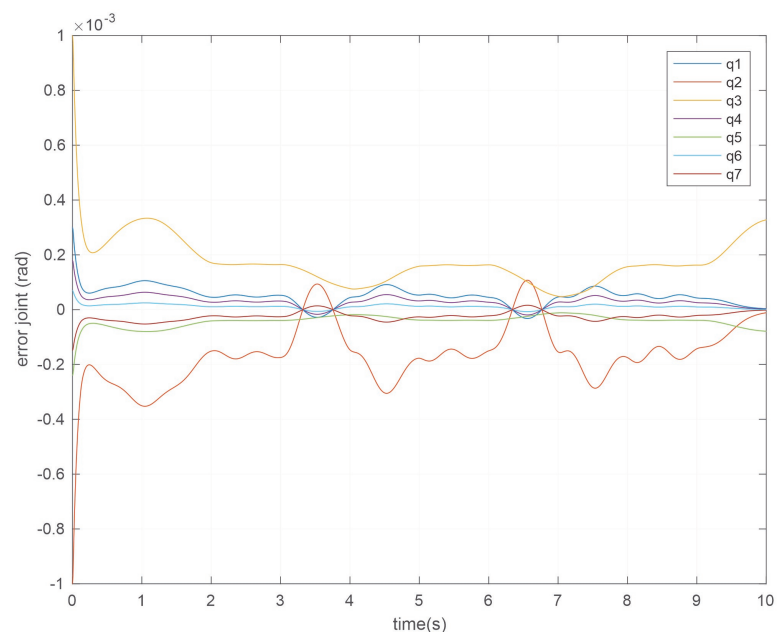

Figure 4. Tracking error of TDVDC

\subsection{Discussion}

The goal of the proposed controller is to track the desired trajectory rather than convergence of physically uncertain parameters. For the two numerical simulations conducted using the proposed TDVDC and the conventionnal regressor-based VDC, the robot can track the desired references but with relatively a tracking error joint difference. In order to carry out an overall quantitative evaluation of the used controllers, the following evaluation criteria are adopted: Root mean square (RMS) of the tracking position error, defined by (57):

$$
R M S\left[e_{i}(t)\right]=\sqrt{\frac{1}{T} \int_{0}^{T}\left|e_{i}(t)\right|^{2}, \mathrm{~d} t}
$$

where $e_{i}(t)=q_{c i}-q_{d i}$

Computing time (CT) defined as a period from the start to the end moment of run simulation.

Maximum torque (MT) defined as the largest torque input to the joint actuators.

We can conclude that the proposed TDVDC presents the low tracking error compared to the conventionnal Regressor-based VDC. For the generated torque, the proposed controller present the low MT for the second joint $q_{2}$, comparing to the regressor-based VDC for the same joint. In the otherhand, the CPU time with the proposed VDTDC approach is equals to $2.486009(s)$ and for the conventionnal VDC is equals to $4.721131(\mathrm{~s})$, using MATLAB program. Therefore, in addition to the gain in terms of error the proposed VDTDC approach presents gain in terms of generated torque and also the computation time.

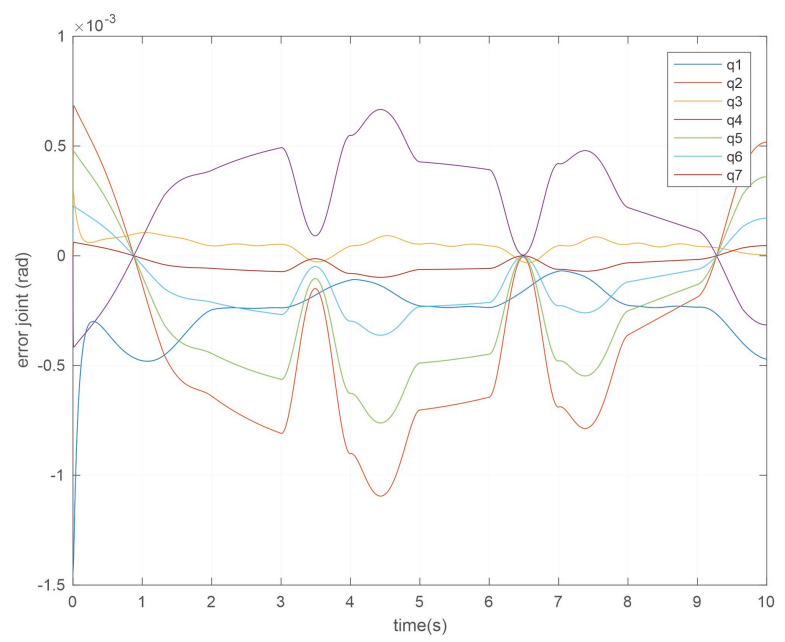

Figure 5. Tracking error of Regressor-based VDC 


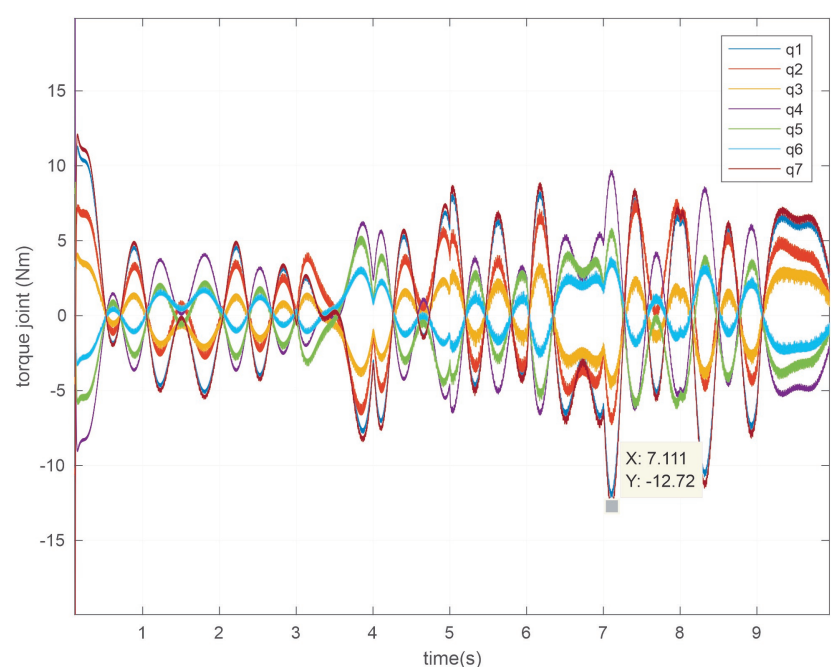

Figure 6. The obtained joint torques for the VDTDC controller

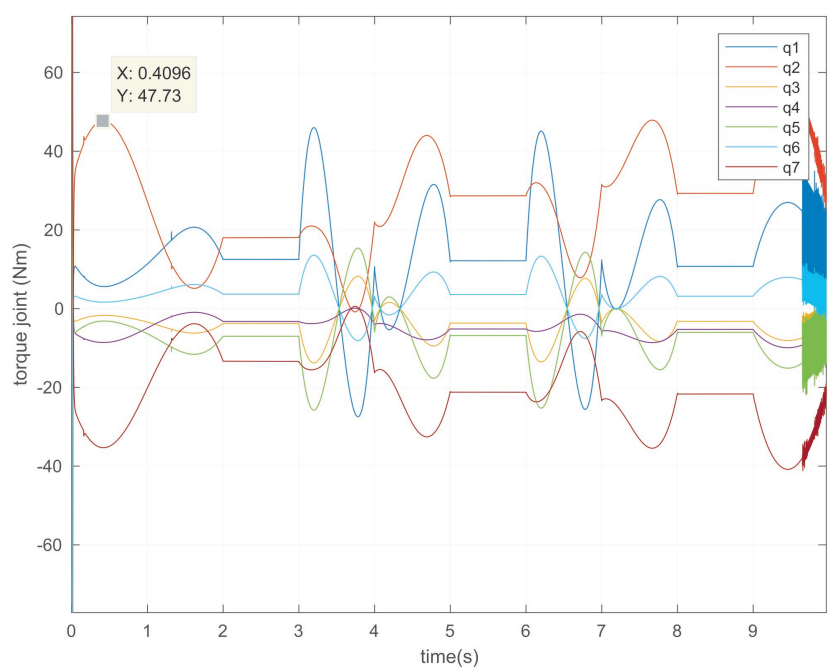

Figure 7. The obtained joint torques for the Regressor-based VDC controller

Table 2. Comparison of RMS errors for regressor-based VDC and VDTDC.

\begin{tabular}{ccr}
\hline Joints & Regressor-based VDC & \multicolumn{1}{c}{ VDTDC } \\
\hline$q_{1}$ & $2.8034^{-04}$ & $0.13^{-04}$ \\
$q_{2}$ & $5.8692 e^{-04}$ & $4.0210^{-04}$ \\
$q_{3}$ & $5.9499^{-04}$ & $1.2010^{-04}$ \\
$q_{4}$ & $3.5726^{-04}$ & $3.5699^{-05}$ \\
$q_{5}$ & $4.0829^{-04}$ & $4.6723^{-05}$ \\
$q_{6}$ & $1.9394^{-04}$ & $1.3883^{-05}$ \\
$q_{7}$ & $5.2568^{-04}$ & $2.0904^{-04}$ \\
\hline
\end{tabular}

\section{CONCLUSION}

To sum up, in this paper, a new aproach has been developed of nonlinear control of n-DoF robot manipulator. The proposed controller is a modified VDC introducing the TDE technique to estimate unknown and uncertainties vector parameters. The system modeling, and the VDTDC controller design were presented. Hence, the stability of overall system was proved based on the Lyapunov theory. Simulation results performed with 7-DoF robot manipulator for the proposed TDVDC controller, shows the capability of the controller for 
tracking the predefined trajectory. Comparatively to the conventionnel regressor-based VDC, the TDVDC presents the lower tracking error, with the lower value of maximum torque, and finally lower computation time, wich proves the effectiveness of the propsal. As futur work, the proposed controller were be tested experimentally to another kind of application.

\section{REFERENCES}

[1] L.Zhijun, Y.Chenguang, and T. Yong, "Decentralised adaptive fuzzy control of coordinated multiple mobile manipulators interacting with non-rigid environments," IET Control Theory and Applications, vol.3, no.7, pp. 397410, 2013, doi:10.1049/iet-cta.2011.0334.

[2] M. Rahmani, H.Komijani, and M.H.Rahman, "New Sliding Mode Control of 2-DOF Robot Manipulator Based on Extended Grey Wolf Optimizer," International Journal of Control, Automation, and Systems, vol. 18, no. 6, pp.1572-1580, 2020, doi:10.1007/s12555-019-0154-x.

[3] W.M.Spong, S.Hutchinson, and M.Vidyasagar, Robot modeling and control," John Wiley and Sons., 2006.

[4] H.Faqihi, M.Saad,K.Benjelloun, M.Benbrahim, M.N.Kabbaj, "Tracking trajectory of a cable-driven robot for lower limb rehabilitation," International Journal of Electrical, Computer, Energetic, Electronic and Communication Engineering, vol. 6, no. 8, 2016, doi: 10.5281/zenodo.1125771.

[5] E.Dombre, and W.Khalil, Robot Manipulators: Modeling, Performance Analysis and Control, Wiley ISTE, 2007.

[6] J.Slotine, and W.Li, Applied nonlinear control, Printice-Hall international, 1991.

[7] X.Liu, G.Gu, and K.Zhou, "Robust stabilization of mimo nonlinear systems by backstepping," Automatica, vol.35, no. 5, pp. 987 992, 1999, doi: 10.1016/S0005-1098(98)00236-2.

[8] S.T.Venkataraman, and S.S.Gulati, "Control of nonlinear systems using terminal sliding modes," ASME. Journal of Dynamic Systems, Measurement, and Control, vol. 115, no. 3, pp.554560, 1993, doi: 10.1115/1.2899138.

[9] S.A. Ajwad , J.Iqbal, M.Imran Ullah, and A.Mehmood , "A systematic review of current and emergent manipulator control approaches," Front. Mech. Eng, vol. 10, pp. 198-210, 2015, doi: 10.1007/s11465015-0335-0.

[10] C. Y. Kuo, , and S.-P. T. Wang, "Nonlinear robust industrial robot control," Journal of Dynamic Systems, Measurement, and Control, vol.111, no. 1, pp. 24-30, 1998, doi: 10.1115/1.3153015.

[11] M. Rahmani, and M.H. Rahman, "Adaptive Neural Network Fast Fractional Sliding Mode Control of a 7-DOF Exoskeleton Robot," International Journal of Control, Automation, and Systems, vol. 18, pp.124133, 2020, doi: 10.1007/s12555-019-0155-1.

[12] Y. Kali, M.Saad, K.Benjelloun, and C.Khairallah, "Super twisting algorithm with time delay estimation for uncertain robot manipulators," Nonlinear Dynamics, vol. 93, no. 2, pp: 557-569, Jul. 2018, doi: 10.1007/s11071-018-4209-y.

[13] J.,Koivumäki, and J.Mattila, "Stability guaranteed impedance control of hydraulic robotic manipulators," IEEE Trans. Mechatronics, vol. 22, no. 2, pp. 601-612, April 2017, doi: 10.1109/TMECH.2016.2618912.

[14] L.Bascetta, G.Ferretti, and B.Scaglioni, "Closed form newton-euler dynamic model of flexible manipulators," Robotica, vol. 35, no. 5, pp. 1006-1030, 2017, doi:10.1017/S0263574715000934.

[15] S. Yang, B.Xia, X.Wang, H.Liu, and B.Liang, "Virtual Decomposition Control of a 3 DOFs Exoskeleton Robot for Space Teleoperation," 2017 IEEE International Conference on Information and Automation (ICIA), 2017, pp. 7-11, doi: 10.1109/ICInfA.2017.8078874.

[16] C. Ochoa-Luna, M.H.Rahman, M.Saad, P. Archambault, and W-H.Zhu, "Virtual decomposition control of an exoskeleton robot arm," Robotica, vol. 34, no. 7, pp. 1587-1609, 2016, doi: 10.1017/S026357471400246X.

[17] W.Zheng, and M.Chen, "Tracking control of manipulator based on high-order disturbance observer," IEEE Access, vol. 6, pp. 26753-26764, 2018, doi: 10.1109/ACCESS.2018.2834978.

[18] M.Galicki, "Finite-time trajectory tracking control in a task space of robotic manipulators," Automatica, vol.67, pp. 165-170, 2016, doi: 10.1016/j.automatica.2016.01.025.

[19] H.H.Asada , F.E.Sotiropoulos, "Dual faceted linearization of nonlinear dynamical systems based on physical modeling theory," J. Dyn. Sys., Meas., Control, vol. 141, no.2, p.021002, 2019, doi: $10.1115 / 1.4041448$. 
[20] Y. Wang, S.Jiang, B.Chen, and H.Wu, "Trajectory tracking control of underwater vehicule-manipulator system using discrete time delay estimation," IEEE Access, vol. 5, pp. 7435-7443, 2017, doi: 10.1109/ACCESS.2017.2701350.

[21] W.-H. Zhu, Virtual Decomposition Control: Toward Hyper Degrees of Freedom Robots, Springer-Verlag, Berlin, Heidelberg, 2010.

[22] H.Liu, and T. Zhang, "Fuzzy sliding mode control of robotic manipulators with kinematic and dynamic uncertainties," Journal of Dynamic Systems, Measurement, vol. 134, no. 6, p. 061007, 2012, doi: 10.1115/1.4006626.

[23] I.Imura, T.Sugie, and T. Yoshikawa, "Adaptive robust control of robot manipulators-theory and experiment," IEEE Transactions on Robotics and Automation, vvol. 10, no. 5, pp. 705-710, Oct. 1994, doi: $10.1109 / 70.326574$.

[24] G.Tao, "Adaptive control of partially known systems," IEEE Transactions on Automatic Control, vol. 40, no. 10, pp. 1813-1818, Oct. 1995, doi: 10.1109/9.467665.

[25] Y.Zhu, and, P.Pagilla, "Adaptive controller and observer design for a class of nonlinear systems,"J. Dyn. Sys., Meas., Control, vol.128, no.3, pp. 712-717, 2006, doi: 10.1115/1.2234489.

[26] H.Liu, S.Li and B.Wang, "Virtual decomposition controller for flexible-joint robot manipulators with nonfull-state feedback". International journal of advanced robotic systems, vol. 14, no. 6, pp. 1-7, 2017, doi: $10.1177 / 1729881417745676$.

[27] B. Brahmi, M. Saad, C. Ochoa-Luna, M. H. Rahman and A. Brahmi, "Adaptive Tracking Control of an Exoskeleton Robot With Uncertain Dynamics Based on Estimated Time-Delay Control," in IEEE/ASME Transactions on Mechatronics, vol. 23, no. 2, pp. 575-585, April 2018, doi: 10.1109/TMECH.2018.2808235.

[28] M. Jin, S. H. Kang, P. H. Chang, and J. Lee, "Robust Control of Robot Manipulators Using Inclusive and Enhanced Time Delay Control," in IEEE/ASME Transactions on Mechatronics, vol. 22, no. 5, pp. 2141-2152, Oct. 2017, doi: 10.1109/TMECH.2017.2718108.

[29] Y. Wang, S.Jiang, B.Chen, and H.Wu, "Trajectory tracking control of underwater vehicule-manipulator system using discrete time delay estimation," IEEE Access, vol. 5, pp. 7435-7443, 2017, doi: 10.1109/ACCESS.2017.2701350.

[30] Y.. Wang, J.Peng, W. Sun, H.Yu, and H.Zhang, "Robust adaptive tracking control of robotic systems with uncertainties," Journal of Control Theory and Applications, vol.6, no. 3, pp. 281286, 2008, doi: 10.1007/s11768-008-6147-6.

[31] D.Braganza, W.E.Dixo, D. M. Dawson, and B. Xian, "Tracking control for robot manipulators with kinematic and dynamic uncertainty," Int. J. Robotics and Automation, vol.23, no.2, pp. 117-126, 2008, doi: $10.5555 / 1750496.1750502$.

[32] H.Liu, and T.Zhang, "Fuzzy sliding mode control of robotic manipulators with kinematic and dynamic uncertainties," Journal of Dynamic Systems, Measurement, and Control, vol.134, no.6, p. 061007, 2012, doi: 10.1115/1.4006626.

[33] H.Al-Shuka, R.Song, "Hybrid regressor and approximation-based adaptive control of robotic manipulators with contact-free motion," 2018 2nd IEEE Advanced Information Management,Communicates,Electronic and Automation Control Conference (IMCEC), 2018, pp. 325-329, doi: 10.1109/IMCEC.2018.8469628.

[34] H.Al-Shuka, B.Corves, and W-H. Zhu, "Function approximation technique-based adaptive virtual decomposition control for a serial-chain manipulator," Robotica, vol.32, pp.375-399, 2014, doi: 10.1017/S0263574713000775.

[35] A. Huang and M.-C.Chien,Adaptive Control of Robot Manipulators: A Unified Regressor-Free Approach, worldscientific, 2010, doi:10.1142/7760.

[36] M. Jin, S. H. Kang, and P. H. Chang, "Robust Compliant Motion Control of Robot With Nonlinear Friction Using Time-Delay Estimation," in IEEE Transactions on Industrial Electronics, vol. 55, no. 1, pp. 258-269, Jan. 2008, doi: 10.1109/TIE.2007.906132.

[37] Y. K.Toumi, and O.Ito, "A time delay controller for systems with unknown dynamics," (ASME) Journal of Dynamic System, Measurement ans Control, vol. 112, no. 1, pp.133-141, 1990, doi: 10.1115/1.2894130.

[38] T. Hsia and L. Gao, "Robot manipulator control using decentralized linear time-invariant time-delayed joint controllers," Proceedings., IEEE International Conference on Robotics and Automation, 1990, 1990, pp. 2070-2075 vol.3, doi: 10.1109/ROBOT.1990.126310. 